\title{
Stereotyped distribution of midbody remnants in early C. elegans embryos requires cell death genes and is dispensable for development
}

Cell Research (2014) 24:251-253. doi:10.1038/cr.2013.140; published online 15 October 2013

\section{Dear Editor,}

The midbody is a structure formed within the intercellular bridge towards the end of cytokinesis [1]. Microtubules within this bridge are then severed on one side of the midbody during abscission, thus generating a midbody remnant in one of the resulting daughter cells. Midbody remnants persist long after cell division and accumulate preferentially in stem cells, induced pluripotent stem (iPS) cells, and cancer stem cells [2-4]. Upon induction of differentiation, midbody remnants are degraded by autophagy or released into the extracellular milieu in some tissue culture cells, and it has been proposed that such removal is critical for enabling a differentiation program [2-4]. However, the fate of midbody remnants in a developing organism remains elusive, and whether their presence plays a role in cell fate determination in vivo is not known.

We addressed these questions in early Caenorhabditis elegans (C. elegans) embryos. We performed time-lapse differential interfering contrast (DIC) and fluorescence imaging to track the midbody remnant generated during the cleavage of the zygote $\mathrm{P}_{0}$ into the anterior blastomere $\mathrm{AB}$ and the posterior blastomere $\mathrm{P}_{1}$ (Figure $1 \mathrm{~A}$ and $1 \mathrm{~B}$ ). We also tracked the midbody remnants generated during the subsequent cleavages of $\mathrm{AB}$ into $\mathrm{ABa}$ and $\mathrm{ABp}$, as well as of $\mathrm{P}_{1}$ into EMS and $\mathrm{P}_{2}$ (Figure $1 \mathrm{~A}$ ). We used GFP-tagged ZEN-4 to track midbody remnants, and either DIC or mCherry fused to a pleckstrin homology (PH) domain to visualize the periphery of cells [5]. Unexpectedly, we found that whereas the $\mathrm{P}_{0}$ midbody remnant is present initially between the $\mathrm{AB}$ and $\mathrm{P}_{1}$ blastomeres (Figure $1 \mathrm{C}-0 \mathrm{~min}$ and $1 \mathrm{D}-0 \mathrm{~min}$, red arrowheads), it is then almost invariably internalized into the EMS blastomere or one of its immediate descendants (Figure 1B, 1C-15 min and 1D-20 min, red arrows, Supplementary information, Figure S1A, S1B, Movies S1 and S2). The $\mathrm{P}_{0}$ midbody remnant is internalized and contains membranous material, as evidenced by the presence of mCherry-PH and the early endosomal marker GFP::RAB-5 (Supplementary information, Figure S1C-S1D), which is consistent with the fact that the midbody is internalized into the C. elegans Q neuroblast lineage later in development [5]. Furthermore, we found that the $\mathrm{AB}$ midbody remnant is also usually internalized by the EMS lineage in the early embryo (Figure 1C-20min and 1D-20 min, purple arrows, Supplementary information, Figure S1A, S1B, S2G and Movie S1). We also found that the $\mathrm{P}_{1}$ midbody remnant is typically internalized by the $\mathrm{P}_{2}$ blastomere (Figure 1C-20 min, yellow arrow, Supplementary information, Figure S1A, S1B and Movie S1). We conclude that midbody remnants have a stereotyped distribution in early C. elegans embryos (Figure 1A).

We next investigated the mechanisms directing the internalization of the $\mathrm{P}_{0}$ and $\mathrm{AB}$ midbody remnants almost exclusively into the EMS lineage and not into $\mathrm{ABa}$ or $\mathrm{ABp}$, which are also in contact with these midbody remnants. We first tested whether such stereotyped distribution is under the control of anterior-posterior (A-P) polarity cues. We used RNAi to deplete par-2 and par3 , which are essential for the A-P polarity, and found that the $\mathrm{P}_{0}$ and $\mathrm{AB}$ midbody remnants are internalized indiscriminately by either an anterior or posterior blastomere in such embryos (Figure 1B, Supplementary information, Figure S2C, S2D, S2F and S2G). Therefore, A-P polarity contributes to directing midbody remnant segregation in early C. elegans embryos.

What aspect of A-P polarity is relevant for the stereotyped internalization of midbody remnants observed in the wild-type? In the one-cell embryo, A-P polarity cues ensure that a larger net pulling force acts on the posterior spindle pole, thus enabling asymmetric spindle positioning and an unequal first cleavage [6]. We reasoned that this might impart a bias during abscission, so that the $\mathrm{P}_{0}$ midbody remnant is positioned closer to the $\mathrm{P}_{1}$ blastomere and thus gets internalized by its descendants of the EMS lineage. We set out to test this hypothesis by depleting the partially redundant $\mathrm{G} \alpha$ proteins GOA-1/GPA-16; 
A

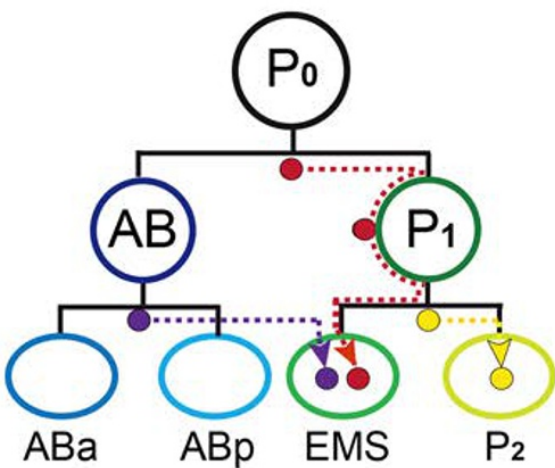

B

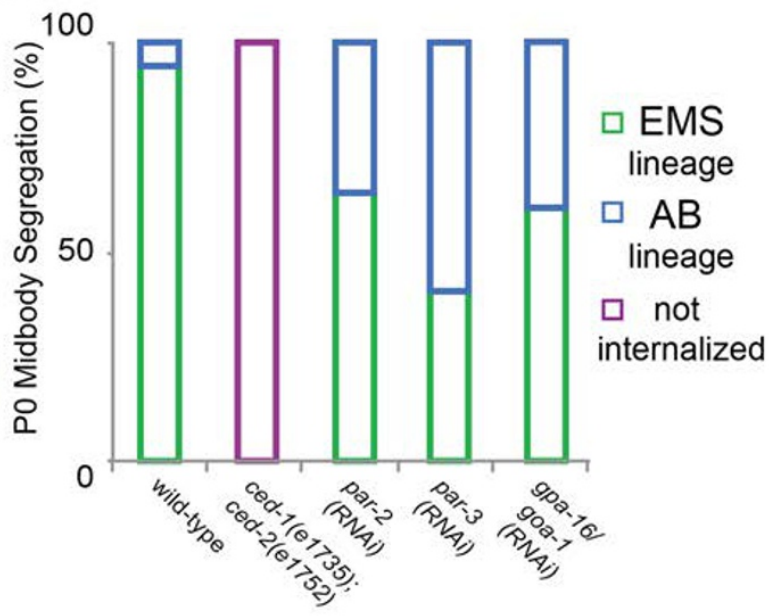

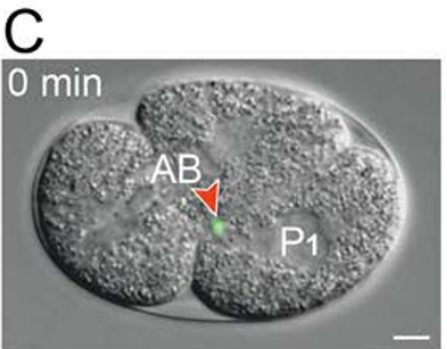
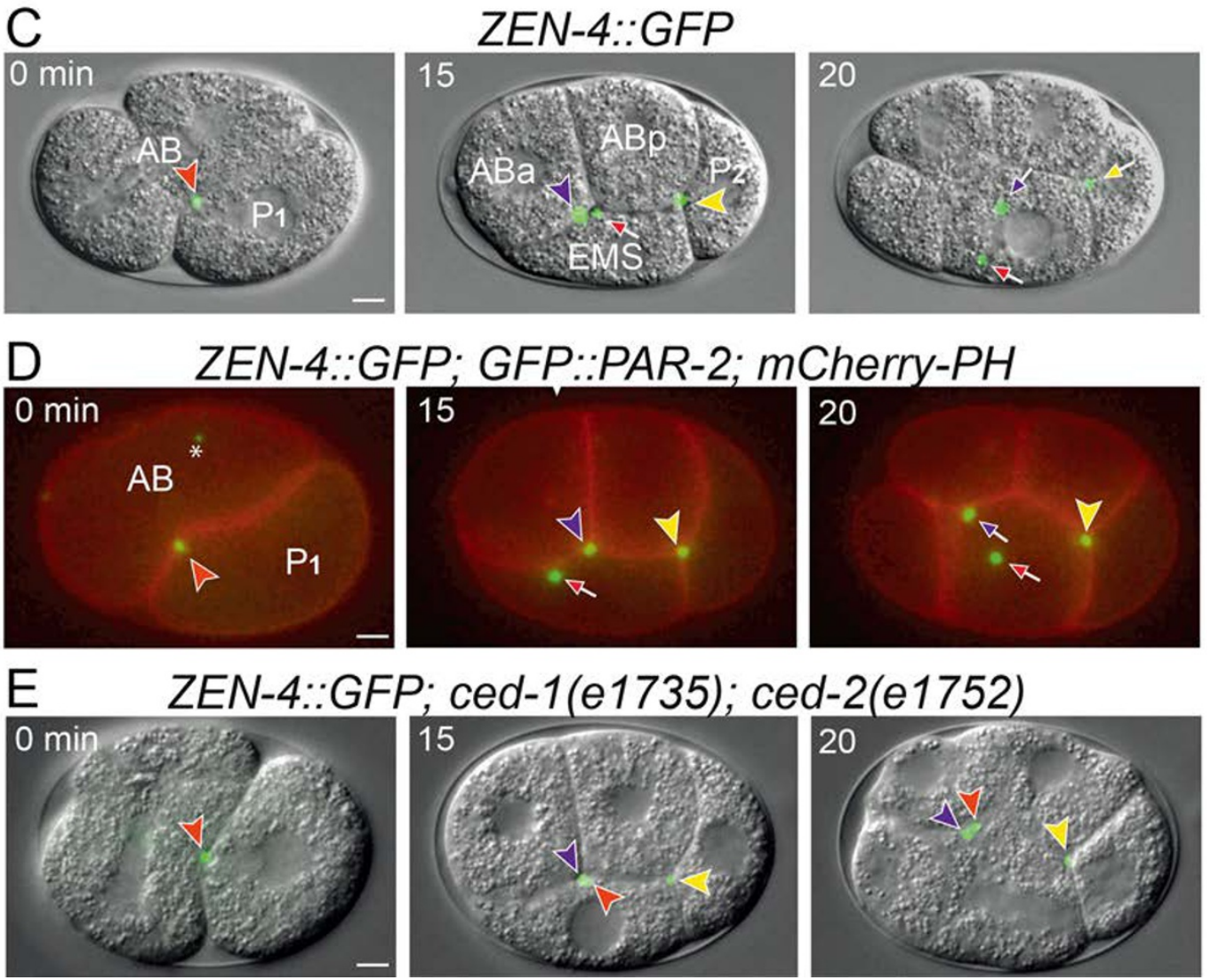

Figure 1 Stereotyped distribution of midbody remnants in early C. elegans embryos. (A) Schematics of the distribution of midbody remnants in early C. elegans embryos, along with the name of blastomeres. Color code of midbody remnants is as in C-E. (B) Distribution of the $\mathrm{P}_{0}$ midbody remnant in embryos of the indicated genotypes. Color code of blastomeres is as in $\mathbf{A}$. Wild-type, $N=18$; ced-1(e1735); ced-2(e1752), $N=10$; par-2(RNAi), $N=11$; par-3(RNAi), $N=16$; goa-1/gpa-16(RNAi), $N=10$. Note that the identity of blastomeres is altered in par-2(RNAi) and par-3(RNAi) embryos, hence our referring to them as "anterior" (corresponding to $\mathrm{ABa}$ and $A B p$ in the wild type) and "posterior" (corresponding to EMS and $P_{2}$ in the wild type) in the main text. (C, E) Images from dual DIC and time-lapse fluorescence microscopy of otherwise wild-type (C) or ced-1(e1735); ced-2(e1752) double-mutant embryos (E) expressing ZEN-4::GFP. The GFP signal has been overlaid onto the DIC image. See also Supplementary information, Movies S1, S2 and S3. (D) Images from time-lapse fluorescence microscopy of wild-type embryo expressing ZEN-4::GFP, GFP::PAR-2 and mCherry- $\mathrm{PH}$. In $\mathrm{C}-\mathrm{E}$, midbody remnants are indicated initially by arrowheads $\left(\mathrm{P}_{0}\right.$ : red, $\mathrm{AB}$ : purple, $\mathrm{P}_{1}$ : yellow). Similarly colored arrows indicate internalization of the corresponding midbody remnant. Blastomere names are indicated in the first two panels in $\mathbf{C}$. Asterisk in $\mathbf{D}$ indicates polar body. Scale bars: $5 \mu \mathrm{m}$. Time is indicated in minute, starting from the onset of AB cytokinesis. 
this results in one-cell embryos undergoing symmetric spindle positioning despite proper A-P polarity [7]. Remarkably, we found that goa-1/gpa-16(RNAi) embryos exhibit randomized segregation of the $\mathrm{P}_{0}$ midbody remnant (Figure 1B and Supplementary information, Figure $\mathrm{S} 2 \mathrm{E}$ ). Distribution of the $\mathrm{AB}$ and $\mathrm{P}_{1}$ midbody remnants is randomized as well in such embryos (Supplementary information, Figure $\mathrm{S} 2 \mathrm{G}$ and $\mathrm{S} 2 \mathrm{H}$ ), perhaps as a consequence of the equal first division. Overall, we conclude that the stereotyped distribution of the $\mathrm{P}_{0}$ midbody remnant, as well as that of the $\mathrm{AB}$ and $\mathrm{P}_{1}$ midbody remnants, albeit perhaps indirectly, are due to the asymmetric positioning of the first mitotic spindle.

We investigated the mechanisms that permit the internalization of midbody remnants in early $C$. elegans embryos. The fact that the AB midbody remnant is incorporated into a cell from the EMS lineage, and not into one from the $\mathrm{AB}$ lineage, implies that it has been released into the extracellular milieu before being internalized. Midbody remnants are also released from C. elegans $\mathrm{Q}$ neuroblasts before being degraded by the neighboring phagocytic cells; the apoptotic cell corpse engulfment genes ced-1 and ced-2 are essential for this process [5]. Since ced-1 and ced-2 are expressed in C. elegans early embryos (see http://nematode.lab.nig.ac.jp/), we examined whether these genes are required for the engulfment of midbody remnants in the embryo. Importantly, we found that the $\mathrm{P}_{0}, \mathrm{AB}$ and $\mathrm{P}_{1}$ midbody remnants remain outside of cells in ced-1, ced-2 mutant or ced-1; ced-2 double-mutant embryos (Figure 1B, 1E, red, purple and yellow arrowheads, Supplementary information, Figure S2A, S2B, S2F, S2G, S2H and Movie S3). We conclude that ced-1 and ced-2 are essential for the internalization of midbody remnants in early $C$. elegans embryos.

In conclusion, we uncovered a remarkably stereotyped segregation of midbody remnants in early $C$. elegans embryos. We showed that such segregation relies on a twotiered mechanism, whereby midbody remnants are first shed into the extracellular milieu in a biased manner due to asymmetric spindle positioning, and then are internalized in a ced-1; ced-2-dependent manner. Importantly, it has been known for decades that ced-1, ced-2 or ced-1; ced-2 mutant animals are viable and do not exhibit obvious defects [8], which we have confirmed by examining ced-1; ced-2 double-mutant embryos expressing ZEN4::GFP (data not shown). Therefore, our findings suggest that midbody remnants are dispensable for fate determination in an intact organism.

\section{Acknowledgments}

GO, CG and PG designed the project and analyzed the data; GO performed most experiments and prepared the figures; $\mathrm{CG}$ performed experiments, prepared the figures and movies; GO and PG wrote the manuscript. We thank Virginie Hachet and Fernando R Balestra for useful comments on the manuscript, as well as Michael Glotzer for the gift of ZEN-4 antibodies. We also thank Olivier Burri and Romain Guiet from the Bioimaging and Optics Platform (BIOP) at EPFL for their help with elaboration of movies. This work was supported by a grant to GO and PG from the Sino Swiss Science and Technology Cooperation (SSTC EG09 092011), and grants from the National Basic Research Program (2012CB945002 and 2013CB945600) and the National Natural Science Foundation of China (31222035, 31190063 and 31171295).

\section{Guangshuo $\mathrm{Ou}^{1,2, *}$, Christian Gentilii, ${ }^{3, *}$ Pierre Gönczy ${ }^{3}$}

\footnotetext{
${ }^{I}$ National Laboratory of Biomacromolecules, Institute of Biophysics, Chinese Academy of Sciences, 15 Datun Road, Chaoyang District, Beijing 100101, China; ${ }^{2}$ Tsinghua-Peking Center for Life Sciences, School of Life Sciences, Tsinghua University, Beijing 100101, China; ${ }^{3}$ Swiss Institute for Experimental Cancer Research (ISREC), School of Life Sciences, Swiss Federal Institute of Technology Lausanne (EPFL), Lausanne CH-1015, Switzerland

*These two authors contributed equally to the work. Correspondence: Pierre Gönczy ${ }^{\mathrm{a}}$, Guangshuo $\mathrm{Ou}^{\mathrm{b}}$

åmail: Pierre.Gonczy@epfl.ch

${ }^{b}$ E-mail: guangshuo.ou@gmail.com
}

\section{References}

1 Glotzer M. Nat Rev Mol Cell Biol 2009; 10:9-20.

2 Ettinger AW, Wilsch-Brauninger M, Marzesco AM, et al. Nat Commun 2011; 2:503.

3 Kuo TC, Chen CT, Baron D, et al. Nat Cell Biol 2011; 13:1214-1223.

4 Schink KO, Stenmark H. Curr Biol 2011; 21:R958-R960.

5 Chai Y, Tian D, Yang Y, et al. J Cell Biol 2012; 199:1047-1055.

6 Grill SW, Gönczy P, Stelzer EH, et al. Nature 2001; 409:630-633.

7 Gotta M, Ahringer J. Nat Cell Biol 2001; 3:297-300.

8 Conradt B, Xue D. Programmed cell death. WormBook 2005:1-13.

(Supplementary information is linked to the online version of the paper on the Cell Research website.) 